\title{
KONDE - EIN NETZWERK BRINGT FORSCHUNGS- UND GLAM-INSTITUTIONEN ZUSAMMEN. EIN PROJEKTBERICHT
}

\section{von Johannes Stigler und Helmut W. Klug}

Zusammenfassung: Seit Frühjahr 2017 läuft als Hochschulraum-StrukturmittelProjekt das „Kompetenznetzwerk Digitale Edition“ (KONDE), das sich den Prämissen der Open Science-Community verpflichtet fühlt und darauf zielt, Voraussetzungen für die Etablierung einer nachhaltigen, nationalen Publikationsplattform für Digitale Editionen (DE) für geistes-, sozial- und kulturwissenschaftliche Disziplinen (GSK) zu schaffen. Beiträger*innen der Universitäten Graz, Innsbruck, Klagenfurt, Salzburg und Wien, sowie der Technischen Universität Graz und der Kunstuniversität Graz, gemeinsam mit Fachkolleg*innen der Österreichischen Nationalbibliothek, der Österreichischen Akademie der Wissenschaften und des Adalbert Stifter-Instituts des Landes Oberösterreich haben sich das Ziel gesetzt, eine Forschungsinfrastruktur für Digitale Editionen aufzubauen und österreichweit vorhandene Kompetenzen in diesem Bereich zu bündeln. Diese Infrastruktur soll keinesfalls nur als Hardware-Lösung oder Sammlung technischer Lösungsansätze verstanden werden, sondern möchte das Thema DE auch aus methodisch-theoretischer Sicht systematisch aufarbeiten und die Arbeits- und Diskussionsergebnisse der einschlägigen Scientific Community in einem interaktiven Weißbuch „Digitale Edition" zur Verfügung stellen.

Schlagwörter: Digitale Edition; Open Science; Nachhaltigkeit; Digital Humanities

\section{KONDE - A NETWORK BRINGS TOGETHER RESEARCH AND GLAM INSTITUTIONS. A PROJECT REPORT}

Abstract: The project "Kompetenznetzwerk Digital Edition" (KONDE) is funded by the Federal Ministry of Science, Research and Economy and has been operational since spring 2017. It is committed to the premises of the Open Science Community and aims to create conditions for the establishment of a sustainable publication platform for digital editions (DE). Contributors from the Universities Graz, Innsbruck, Klagenfurt, Salzburg and Vienna, the Graz University of Technology and the University of Arts Graz together with colleagues from the Austrian National Library, the Austrian Academy of Sciences and the Adalbert Stifter Institute of the Province of Upper Austria, have set themselves the goal of establishing a research infrastructure for digital editions and pooling their expertise in this field throughout Austria. This infrastructure should by no means be understood merely as a 
hardware solution or a collection of technical solutions. It will also approach the topic of DE from a methodological-theoretical point of view and make the work and discussion results available to the scientific community in an interactive white paper on digital editing.

Keywords: Digital Scholarly Edition; Open Science; Sustainability; Digital Humanities

DOI: https://doi.org/10.31263/voebm.v72i2.3048

(C) Johannes Stigler, Helmut W. Klug

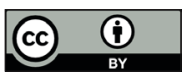

Dieses Werk ist lizenziert unter einer

Creative-Commons-Lizenz Namensnennung 4.0 International

\section{Inhalt}

\section{Einleitung}

2. Ausgangslage

3. Der Digital Turn in der Editionswissenschaft

4. Standardisierung als Akzeptanzkriterium des digitalen Weges

5. Zielsetzung des Projektes

6. Vom Innovation Follower zum Innovation Leader

7. Weiterentwicklung von GSK-Forschung als strategisches Ziel

\section{Ausgangslage}

Editionen als die Erschließung und Verfügbarmachung von Quellen zielen im traditionellen Verständnis vorwiegend auf Texte. Digitale Editionen (DE) im Speziellen sind ein Produkt der Disziplin der Digital Humanities (DH), in der computergestützte Methoden zur Erstellung, Erforschung und Verbreitung von wissenschaftlich fundierten Quellenveröffentlichungen herangezogen werden. Zeichnet sich schon die „klassische“ Edition durch historisch gewachsene Diversität aus, erhält dieses Forschungsgebiet durch die Erweiterung um den Aspekt der digitalen Bearbeitung eine weitere Dimension an Komplexität: Neben den wissenschaftshistorisch betroffenen Fachwissenschaften (Editionsphilologie, Historische Hilfswissenschaften) diskutieren und beeinflussen nun auch andere Forschungsbereiche (z.B. Layout- und Texterkennung, Datenvisualisierung, Historische Fachinformatik, Computerlinguistik) die Entwicklung und Umsetzung von digitalen Editionen. Die Diskus- 
sionen rund um diese Thematik widmen sich nicht nur der Frage um das Verhältnis von Quelle und Edition, sondern reflektieren ganz gezielt auch den Mehrwert, den die Digitalität einer Edition erzeugt. DE sind damit eines der grundlegenden Forschungsgebiete in den digitalen Geisteswissenschaften und darüber hinaus als Querschnittsmethode von größter Relevanz für deren interdisziplinären Charakter. In diesem Sinne eröffnen DE Forschungsfragen, die über rein fachwissenschaftliche Fragestellung hinausgehen bzw. diese ergänzen. DE sind also auch an informationswissenschaftliche Fragestellungen gebunden, wobei hier das Verhältnis von Information und Wissen zur Benutzbarkeit einer Edition im Mittelpunkt steht.

\section{Der Digital Turn in der Editionswissenschaft}

Die Diskussion rund um die Bedeutung des Digital Turn in der „klassischen" Editionswissenschaft begann in den 1980er Jahren und oszillierte zwischen radikalen Vorstellungen der völligen Remodellierung der Editionsmethodologie durch Werkzeuge der Computerwissenschaften und einer Verfeinerung der etablierten wissenschaftlichen Methoden mithilfe von digitalen Werkzeugen ${ }^{1}$. Sahle hat die Auswirkungen der neuen Methoden auf das Konzept von Text (als Bezugspunkt jeglicher historisch-kritischen Edition) hervorgehoben ${ }^{2}$ und theoretische Reflexionen darüber angeregt, auf welche Art und Weise DE auch neue Formen der Materialität von Text ${ }^{3}$ zu konstituieren in der Lage sind. In der theoretischen Diskussion rund um die Digitalisierung der Geisteswissenschaft wird meist zwischen artefaktorientierten Disziplinen (z.B. Archäologie) und textorientierten Disziplinen (z.B. Literaturwissenschaft) unterschieden. Textgebundenheit stellt jedoch insgesamt ein konstitutives Element geisteswissenschaftlicher Erkenntnisprozesse dar und so scheint es zulässig, Konzepte der digitalen Bearbeitung von Text nutzbringend auch auf methodologische Probleme artefaktorientierter Disziplinen anzuwenden. Aus dieser Perspektive konstituiert sich die Digitalisierung (= digitale Repräsentation) kultureller Artefakte nicht einfach über deren bildhafte oder textuelle Repräsentation im Computer, sondern auch im Bemühen um die (formale) Erschließung, Annotation und Kontextualisierung der semantischen Strukturen dieser Artefakte. Dabei geht es sowohl um die Sichtbarmachung der dem Artefakt immanenten Semantiken als auch um die Anreicherung mit Bedeutungen, die aus Interpretations- und Kontextualisierungsakten entstehen. 


\section{Standardisierung als Akzeptanzkriterium des digitalen Weges}

Einer der Gründe für den Paradigmenwechsel von „klassischer“ zu DE ist die Veränderung am wissenschaftlichen Arbeitsplatz, die von einer Transmedialisierung gekennzeichnet ist: Alle editorischen Verfahren werden mittlerweile in einer digitalen Arbeitsumgebung an digitalen Dokumenten ausgeführt. Konsequenterweise sollte daher auch das Ergebnis editorischer Arbeit digital sein. Das schafft gleichzeitig die Grundlage für eine Vielzahl an Derivaten, die neben dem gedruckten Lesetext u.a. Text-Bild-Relation, unterschiedliche Textdarstellungsvarianten, textgenetische Dokumentation, Darstellung und Analysen oder die Auswertungen und Visualisierungen der Textdaten und von in den Texten beschriebenen Daten umfassen kann. Eine Voraussetzung dafür ist aber, dass die Variantenvielfalt innerhalb der digitalen Forschungsumgebung zwar ihren experimentellen Charakter beibehält, aber mittelfristig zu generischen Lösungen hingeführt wird. Damit ist nicht nur die Möglichkeit einer vielfältigen Präsentation vorhanden, sondern die erarbeiteten Daten können auch nach den gängigen Richtlinien einer Langzeitarchivierung (LZA) zugeführt werden.

Zurzeit gibt es allerdings neben einigen wenigen Repositorien (Fokus: Archivierung) und Arbeitsplattformen (Fokus: Transkription und Publikation) ausschließlich proprietäre Ansätze zur DE, die bislang geringe Akzeptanz in den Fach- und Editionswissenschaften gefunden haben. Das wird zum ersten durch die Arbeit an Datenaustauschstandards, insbesondere im Rahmen der Text Encoding Initiative $\left(\mathrm{TEI}^{4}\right)$ aber auch im Kontext von Bildreferenzierung $\left(\right.$ IIIF $^{5}$ ) und Metadaten-Harvesting (Canonical Text Services ${ }^{6}$, correspSearch ${ }^{7}$ ), angestrebt. Auch wird vielerorts an Qualitätskriterien für DE gearbeitet, wie es durch das deutsche Institut für Dokumentologie und Editorik ${ }^{8}$ (IDE) im Rahmen seines Rezensionsorgans RIDE ${ }^{9}$, mit den Antragsrichtlinien für literaturwissenschaftliche Editionen der DFG oder Initiativen der MLA vorangetrieben wird. Schließlich bereiten Forscher*innen editionenübergreifende Werkzeuge (CollateX ${ }^{10}$, Pelagios ${ }^{11}$ ) zur Datenanalyse und -aufbereitung vor, die als individuelle Module in die Genese und Präsentation einer DE eingebunden werden können. Ein Desiderat ist also noch immer eine domänen- und disziplinenübergreifende, modular ausgerichtete Forschungsinfrastruktur, in der die Funktionen des Repositoriums auf eine editorische Arbeitsumgebung und Publikationsplattform treffen, in der editionsspezifische Analysemodule nach Bedarf für Forschungsfragen aktiviert werden können. Die Universität Graz als Lead-Institution des Kompetenznetzwerkes war durch die Mitarbeit am Marie-Curie Initial Training Network „DiXiT“12 Teil einer führenden inter- 
nationalen Forscher*innengruppe im Bereich DE und kann außerdem auf langjährige, einschlägige Erfahrung mit dem Geisteswissenschaftlichen Asset Management System ${ }^{13}$ (GAMS) zurückgreifen, in dem eine einheitliche Infrastruktur für die unterschiedlichsten fachwissenschaftlichen Anforderungen verwendet wird.

\section{Zielsetzung des Projektes}

Basierend auf dieser Ausgangslage zielt das Netzwerk auf den Aufbau und die Weiterentwicklung einer nationalen Forschungsinfrastruktur für DE und versteht sich darüber hinaus auch als eine Maßnahme für die Etablierung der Community im Fachbereich Digitale Geisteswissenschaften in Österreich. In neun interdisziplinär besetzten Arbeitsgruppen wird ein Referenzmodell erarbeitet, das die Basis für den Aufbau einer Plattform zur dauerhaften, kuratierbaren Bereitstellung von Digitalen Editionen bilden soll. Arbeitsgruppen zu folgenden Themenbereichen wurden dazu eingerichtet:

- Transkription und Textauszeichnung

- Korpuslinguistische Analyse und Textmining

- Webpräsentationsform und Interfaces

- Alternative Formen der Textauszeichnung

- Netzwerkanalyse und Datamining

- Langzeitarchivierung

- Textkritik und Kommentar

- Hybrid-Edition

Folgende Themenkreise bilden dabei die Kristallisationspunkte der Diskussionen in den einzelnen Arbeitsgruppen:

- die Definition von Workflows und Standards für die systematische und institutionenübergreifende Digitalisierung und Zurverfügungstellung von Quellenmaterial,

- die Entwicklung von Prototypen zur Texteingabe bei der Erfassung handschriftlicher (historischer) Dokumente in Abstimmung mit, aber auch in Erweiterung geltender Standards.

- die (Weiter-)Entwicklung von Werkzeugen für die Verarbeitung digitaler Textkorpora mittels (semi-)automatischer Verarbeitungsschritte (z.B. Texterkennung in Handschriften und Inschriften, heuristische und inferenzstatistische Auffindung und Analyse von semantischen Textstrukturen etc.). 
- den Aufbau und die Etablierung einer einschlägigen Fortbildungsinfrastruktur zur Vermittlung der im Projekt erarbeiteten Standards,

- der Aufbau und die Schaffung von Best-Practice Beispielen von Open Source- und Open Access-Strategien für DE im Sinne zeitgemäßer Open Science-Richtlinien.

\section{Vom Innovation Follower zum Innovation Leader}

Für die Entwicklung Österreichs vom Innovation Follower zu einem Innovation Leader im Bereich der DE ist der Ausbau einer auf Open Science basierenden GSK-Forschungsinfrastruktur unumgänglich. Das Projekt KONDE unterstützt die Grundlagenforschung zu DE, die eine zentrale Rolle in der Erschließung und Bewahrung des kulturellen Erbes einnimmt. Dadurch, dass das Projekt Mitarbeiter*innen aller österreichischen KeyPlayer aus der Forschung sowie aus dem Bibliotheks-, Archiv- und Museumssektor (BAM) zusammenbringt, können einander ergänzende und Synergien erzeugende Maßnahmen beschlossen werden, eine Koordination und Zusammenführung des Forschungsbetriebs stattfinden und die einzelnen Forschungszentren gestärkt werden. Diese Art der Schwerpunktsetzung unterstützt die internationale Positionierung in diesem Forschungsbereich und stärkt auch die universitäre Lehre nachhaltig. Der Aspekt der Nachhaltigkeit spiegelt sich in diesem Projekt in einer konsequenten Open Science-Ausrichtung wieder, die für eine langfristige und freie Verfügbarkeit von Forschungsdaten und Forschungsergebnissen einen maximalen gesellschaftlichen Nutzen durch Open Access-, Open Data- und Open Source Software-Strategien erzielt. Gleichzeitig wird nach gängigen GSKStandards eine Qualitätssicherung der geleisteten Forschung betrieben, das beinhaltet u.a. kontinuierliche interne Methodendiskussion, Präsentation auf einschlägigen Veranstaltungen mit Peer Review-Auswahlverfahren, Publikationen in Peer Review-Publikationen und Rezensionen der entstehenden Editionen in einschlägigen Fachzeitschriften (z.B. RIDE). Die strenge Ausrichtung nach den Open Science-Richtlinien (Open Access, Open Data, Open Methodology) ist ein zentraler Schritt zur Öffnung der Wissenschaft gegenüber der Gesellschaft, die Grundlage für glaubwürdige Wissenschaftskommunikation und die Basis für partizipative Wissenschaft. Das im Projekt zu konzipierende modulare System für DE ermöglicht bei Bedarf und wissenschaftlicher Indiziertheit selbstverständlich auch die aktive Einbindung von Laien im Sinne von Citizen Science Forschungsansätzen. 


\section{Weiterentwicklung von GSK-Forschung als strategisches Ziel}

Der Forschungsaktionsplan des BMWFW ${ }^{15}$ (2015) hat die Weiterentwicklung der GSK-Forschung als ein zentrales Anliegen, weil gesamtstaatliche und -gesellschaftliche Entwicklung und Innovation nur durch das Wissen, die Erfahrung und die Analysen aus diesen Fächern möglich wird: Zentrale Schlagworte dazu sind die Erschließung und die Bewahrung des geistigen und kulturellen Erbes. DE, die unter Einhaltung der Open Science-Vorgaben erstellt werden, wirken unmittelbar auf ihre Rezipient*innen ein. Sie sind die Basis für unterschiedlichste Disseminationsstrategien (medienunabhängig, rezipientenunabhängig, zeitunabhängig). Gleichzeitig spiegeln DE die Veränderungen im Umgang mit der Medialität von Forschungsdaten und -ergebnissen wider, sowie auch einen Wandel in der Selbstwahrnehmung der GSK-Fächer, der vor allem in der Citizen Science-Orientierung, die gerade für DE von Bedeutung ist, gesehen werden kann.

Viele Ziele des vorliegenden Projektes finden sich auch in den Forderungen der $\mathrm{H} 2020^{16}$-Ausschreibungen für eine europäische e-Infrastruktur wieder, allen voran die Maßnahmen gegen eine fragmentierte Forschungslandschaft. Wie in den Projektzielen formuliert, soll einer solchen Fragmentierung durch entsprechende Konzepte und Rollenmodelle entgegengewirkt werden, damit die Forschung, deren Daten und Ergebnisse frei zugänglich und auch für andere Forschungszwecke wiederverwertbar sind. Langzeitarchivierung der Daten ist ebenso eine Zielsetzung wie die Aufbereitung der Daten für maschinelle Verarbeitung. Die Anwendung höchster wissenschaftlicher Standards (z.B. Referenzierbarkeit, Reproduzierbarkeit) ist dabei genauso selbstverständlich wie die Einhaltung von Open ScienceRichtlinien. Viele Projektpartner*innen sind Mitglieder der ESFRI ${ }^{17}$ Landmarks CLARIN ${ }^{18}$ - und DARIAH-ERIC ${ }^{19}$.

Die DE als zentrale Methode der DH kann als Grundlage für jegliche weitere fachspezifische Forschung gesehen werden, gleichzeitig ist sie auch das Ergebnis einer sich im Wandel befindlichen Forschungskultur in den GSK-Fächern, die von einem analogen zu einem digitalen Forschungsansatz wechselt und dabei ein breites Methodenpotenzial erschließt. Das wird gerade aus dem vorliegenden Projekt ersichtlich, wenn divergierende Forschungsfelder unterschiedlicher Institutionen aufeinandertreffen und Experten*innenwissen interdisziplinär nutzbar gemacht wird. BAMEinrichtungen treffen auf Institute der Technischen Universität, auf IT-Einrichtungen und auf Institute der Geisteswissenschaftlichen Fakultäten. Aus allen Forschungsfeldern und Institutionen kommen fachspezifische Methoden, die in einem Kompetenz- und Wissensnetzwerk 'Digitale Edition' 
integriert werden können. Derart gesammelt ergibt sich die Möglichkeit eines befruchtenden interdisziplinären und interinstitutionellen Methodenaustauschs.

\author{
Mag. Dr. Johannes Stigler \\ ORCID: https://orcid.org/0000-0003-0803-1496 \\ Universität Graz, Zentrum für Informationsmodellierung - \\ Austrian Centre for Digital Humanities \\ E-Mail: johannes.stigler@uni-graz.at \\ Mag. Dr. Helmut W. Klug \\ ORCID: https://orcid.org/0000-0002-7461-5820 \\ Universität Graz, Zentrum für Informationsmodellierung - \\ Austrian Centre for Digital Humanities \\ E-Mail: helmut.klug@uni-graz.at
}

1 Peter Robinson: New Directions in Critical Editing, in: Electronic Text: Investigations in Method and Theory. Hg. von K. Sutherland, Oxford 1997, S. 145-172.

2 Patrick Sahle: Digitale Editionsformen. Zum Umgang mit der Überlieferung unter den Bedingungen des Medienwandels, 3 Bände, Norderstedt 2013 (Schriften des Instituts für Dokumentologie und Editorik 7-9).

3 Martin Schubert ( $\mathrm{Hg}$.): Materialität in der Editionswissenschaft (Beihefte zu editio 32), Walter de Gruyter, Berlin \& New York 2010.

4 Text Encoding Initiative - TEl. http://www.tei-c.org

5 International Image Interoperability Framework - Image API. http://iiif. io/api/image

6 Canonical Text Services - https://github.com/cite-architecture/cts_ spec/blob/master/md/specification.md

7 CorrespSearch - http://correspsearch.net

8 Institut für Dokumentologie und Editorik - https://www.i-d-e.de/

9 RIDE. A review journal for digital editions and resources - https:// ride.i-d-e.de/

10 CollateX - Software for collating texual sources - https://collatex.net/

11 Pelagios Commons - http://commons.pelagios.org

12 Digital Scholarly Editions Initial Training Network - http://dixit.unikoeln.de/

13 Geisteswissenschaftliches Asset Management System - http://gams. uni-graz.at 
14 Open Science Network Austria (OANA) - http://oana.at

15 Bundesministerium für Wissenschaft, Forschung und Wirtschaft - bmwfw (2015): Aktionsplan für einen wettbewerbsfähigen Forschungsraum - Maßnahmen des Bundesministeriums für Wissenschaft, Forschung und Wirtschaft zur verstärkten Umsetzung der FTI-Strategie der Bundesregierung in ausgewählten Themenfeldern. https://www. bmbwf.gv.at/fileadmin/user_upload/wissenschaft/publikationen/ forschung/Forschungsaktionsplan_web.pdf

16 Horizon 2020 Framework - https://ec.europa.eu/programmes/horizon2020/en

17 ESFRI Roadmap - https://www.esfri.eu/esfri-roadmap

18 CLARIN - European Research Infrastructure for Language Resources and Technology - https://www.clarin.eu/

19 DARIAH - Digital Research Infrastructure for the Arts and Humanities - https://dariah.eu/ 